\title{
INDICADORES
}

\section{PROPUESTA DE RANKINGS DE UNIVERSIDADES ESPAÑOLAS EN REDES SOCIALES}

\author{
A proposal of rankings of Spanish universities in \\ social networking sites
}

\section{Carmen Zarco, Salvador Del-Barrio-García y Óscar Cordón}

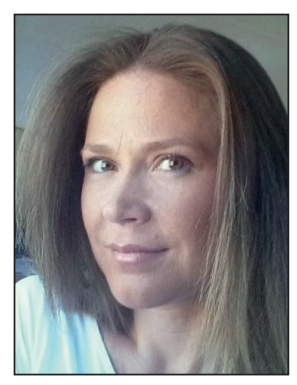

Carmen Zarco es doctora en documentación científica y master en marketing y comportamiento del consumidor por la Universidad de Granada. Su vida profesional se ha desarrollado durante más de quince años en varias empresas como responsable de gestión del conocimiento. A lo largo de su carrera ha publicado una decena de artículos científicos, algunos de ellos en revistas JCR, y ha participado en varios proyectos y contratos de investigación. Es profesora asociada en la Universidad Internacional de La Rioja, donde imparte clase en el grado de ADE y en el MBA. http://orcid.org/0000-0002-2530-249X

Universidad Internacional de la Rioja, Departamento de Comercialización e Investigación de Mercados Gran Vía Rey Juan Carlos I, 41. 26002 Logroño, España carmen.zarco@unir.net

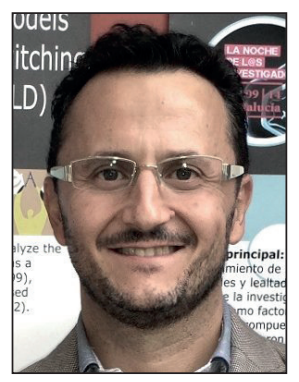

Salvador Del-Barrio-García es profesor titular de comercialización e investigación de mercados en la Universidad de Granada. Sus áreas de especialización son la comunicación integrada de marketing, el comportamiento del consumidor online, marketing cross-cultural y marketing turístico. Ha dirigido 3 tesis doctorales y ha publicado diversos libros y capítulos de libros en editoriales internacionales de prestigio como Springer, Wiley e IGI Global. Ha publicado más de 30 artículos en revistas científicas de prestigio, entre ellas International journal of advertising, Journal of advertising research, European journal of marketing, Crosscultural management: An international journal, Computers in human behaviour, Business services, Cornell hospitality quarterly, Cities, y Public relations review. http://orcid.org/0000-0002-6144-0240

Universidad de Granada, Departamento de Comercialización e Investigación de Mercados Campus Universitario de Cartuja, s/n. 18071, Granada, España dbarrio@ugr.es

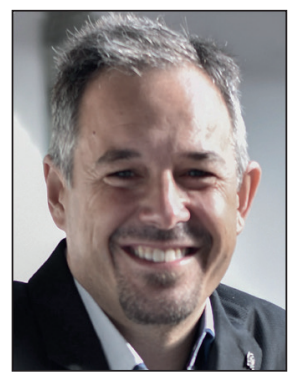

Óscar Cordón, catedrático de la Universidad de Granada, es director del Laboratorio de Soft Computing Applications for Complex EnviRonments (Soccer) y delegado de la Rectora (Vicerrector) para la universidad digital. Ha dirigido 16 tesis doctorales y publicado más de 100 artículos en revistas internacionales, 88 de ellos en revistas JCR. Ha coordinado 26 proyectos y contratos de investigación. Es Premio nacional de informática Aritmel 2014, Premio IEEE computational intelligence society outstanding early career award 2011 y Premio a la trayectoria de jóvenes investigadores de la UGR 2004.

http://orcid.org/0000-0001-5112-5629

Universidad de Granada, Departamento de Ciencias de la Computación e Inteligencia Artificial Periodista Daniel Saucedo Aranda, s/n. 18071, Granada, España ocordon@decsai.ugr.es

\section{Resumen}

La elaboración de rankings es una aproximación cada vez más popular para la evaluación de resultados de las universidades. Las propuestas existentes de rankings de universidades españolas en redes sociales presentan limitaciones como su falta de cobertura, su especificidad y especialmente su falta de transparencia en la metodología de definición. El objetivo de este trabajo es proponer un marco general de diseño de clasificaciones coherentes para analizar el impacto de todas las universidades españolas, tanto públicas como privadas, en varias redes sociales (Twitter, Facebook, Linkedln y YouTube). El 
marco global propuesto está basado en la definición de una taxonomía de criterios, la selección de los disponibles en las cuatro redes sociales mencionadas y la construcción de distintos tipos de indicadores a partir de ellos. Para ilustrarlo, presentamos dos casos de estudio relacionados con la presencia y la influencia de las universidades españolas en dos de esas redes, Twitter y Linkedln.

\title{
Palabras clave
}

Rankings de universidades; Redes sociales; Facebook; Twitter; YouTube; LinkedIn.

\begin{abstract}
University rankings have become popular tools to evaluate the results of academic institutions. The existing proposals of rankings of universities in Spain, based on social networking sites, show some limitations related to their lack of coverage, their specificity, and their lack of transparency in the ranking definition. The goal of this work is to introduce a general design framework for coherent classifications to analyze the impact of all Spanish universities, both public and private, in four social networking sites: Twitter, Facebook, LinkedIn, and YouTube. The proposed framework is based on the definition of a criteria taxonomy, the selection of those criteria available in the four social networking sites, and the proposal of various kinds of indicators using them. To illustrate our framework, two case studies are developed evaluating the presence and influence of Spanish universities in Twitter and LinkedIn.
\end{abstract}

\section{Keywords}

University rankings; Social networking sites; Facebook; Twitter; YouTube; LinkedIn.

Zarco, Carmen; Del-Barrio-García, Salvador; Cordón, Óscar (2016). “Propuesta de rankings de universidades españolas en redes sociales". El profesional de la información, v. 25, n. 4, pp. 684-698.

http://dx.doi.org/10.3145/epi.2016.jul.18

\section{Introducción}

\section{1. redes sociales y universidades}

Los progresos tecnológicos que se han ido produciendo en el campo de la comunicación siempre han sido objeto de investigación dentro de las ciencias sociales al generar nuevas formas de relación social que provocan una transformación en los hábitos y costumbres de la sociedad. Las redes sociales virtuales (en adelante RS) nos permiten interactuar con otras personas aunque no las conozcamos. Estas redes cuentan con una serie de herramientas tecnológicas muy sencillas de utilizar y posibilitan la creación de comunidades de personas en las que se establece un intercambio dinámico y comunicativo (O’Keeffe; Clarke-Pearson, 2011).

Las RS son especialmente adecuadas para alcanzar a uno de los públicos objetivos de mayor interés para las instituciones educativas, los estudiantes, que son los usuarios que más rápidamente las han adoptado

En este contexto, la incursión de las RS supone un salto cualitativo que potencia significativamente los modos de comunicación y generación del conocimiento de los escenarios involucrados, concretamente en la enseñanza (Gonzalo-Brito; Laaser; Toloza, 2012). Las RS son especialmente adecuadas para alcanzar a uno de los públicos objetivos de mayor interés para las instituciones educativas, los estudiantes, que son los usuarios que más rápidamente las han adoptado. Tienen todo el potencial para convertirse en un recurso valioso para conectar e implicar a este colectivo y para ser utilizadas in- cluso como herramientas docentes (Del-Barrio-García; Arquero; Romero-Frías, 2015; Roblyer et al., 2010). Por esta razón, las universidades han sido pioneras en incorporar su uso como herramienta de comunicación y han comenzado a otorgarle más importancia dentro de su estrategia de comunicación institucional (Valerio, 2015). El impacto de las RS en España es muy destacado, siendo uno de los países con mayor tasa de penetración. Concretamente, según datos de la tercera oleada del Estudio general de medios (octubrenoviembre) (EGM 2015), el 64,2\% de la población frecuenta alguna red social (AIMC, 2015). De acuerdo con el informe internacional de GlobalWeblndex, las RS ocupan ya un tercio del tiempo online de los usuarios españoles, los cuales son activos en 3,5 RS de media (GWI, 2016). De todas ellas, las más utilizadas son $(I A B, 2015)^{1}$ :
- Facebook (96\%)
- YouTube (66\%)
- Twitter (56\%)
- Google+ (34\%)
- Linkedln (31\%)

Como consecuencia de este crecimiento en el uso de las RS, especialmente entre los jóvenes, la mayoría de las universidades españolas se han sumado a este movimiento y han abierto perfiles institucionales en los últimos años (Paniagua-Rojano; Gómez-Calderón, 2012; Gómez-Calderón; Paniagua-Rojano, 2014; Simón-Onieva, 2014; González-Díaz; Iglesias-García; Codina, 2015).

\subsection{Rankings de universidades}

Las clasificaciones académicas de universidades o rankings ${ }^{2}$ de universidades han experimentado un gran avance en los últimos años. Estos rankings tienen por objeto realizar jerarquizaciones de las instituciones de educación superior 
teniendo en cuenta diversos indicadores que buscan medir aspectos relacionados con la calidad de la investigación, la docencia u otros aspectos de la actividad académica para informar y orientar a los estudiantes, investigadores y público en general (Liu, 2015; Tomàs-Folch et al., 2015).

Cada vez se tienen más en cuenta como medidas para evaluar la calidad o el prestigio de una universidad. Su estrecha incidencia sobre la opinión pública y su canalización a través de los medios de comunicación permitiría caracterizarlos como una herramienta de marketing que promociona las universidades mejor calificadas (Ordorika; Rodríguez-Gómez, 2010).

Existe un gran número de rankings universitarios de distinta naturaleza a nivel nacional e internacional, muchos de ellos basados en criterios relacionados con la calidad y la cantidad de la producción investigadora. Entre los rankings de universidades del mundo destaca el Academic Ranking of World Universities (ARWU), más conocido como Ranking de Shanghai, considerado actualmente como el referente para conocer la posición de las universidades a nivel mundial (Docampo, 2012; Docampo et al., 2012).

http://www.shanghairanking.com/es

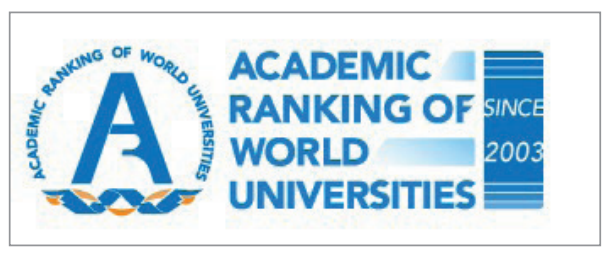

El suplemento educativo de Times, Higher, publica también un ranking mundial anual de universidades desde 2004: $\mathrm{Ti}$ mes Higher Education (THE) World University Rankings. A partir de 2010 comenzó a colaborar con Thomson Reuters utilizando una nueva metodología (Ordorika; RodríguezGómez, 2010).

https://www.timeshighereducation.com/world-universityrankings

Otros rankings internacionales de referencia son:

- QS-Ranking

http://www.topuniversities.com/university-rankings

- SCImago Institutions Rankings

http://www.scimagoir.com

- Leiden Ranking

http://www.leidenranking.com

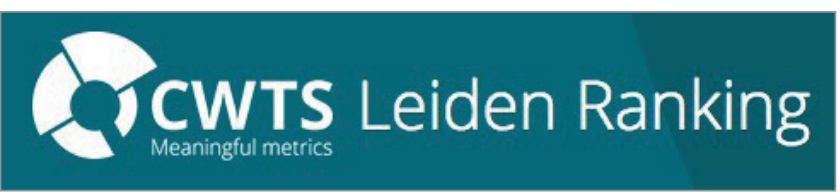
- U-Multirank
http://www.umultirank.org

\section{u multirank \\ Universities compared. Your way.}

- NTU Ranking

http://nturanking.lis.ntu.edu.tw

- URAP

http://www.urapcenter.org/2015

Todos estos rankings se basan principalmente en indicadores de producción científica.

Existen también rankings internacionales que se han centrado en clasificar las universidades en función de su presencia web. En este sentido cabe destacar el Ranking Mundial de Universidades en la Web (Webometrics), una iniciativa del Laboratorio de Cibermetría del CSIC. La intención de este ranking es destacar la importancia de las publicaciones en web como forma de transmisión de conocimiento académico además de medir el rendimiento y el impacto de la actividad científica.

http://www.webometrics.info

En lo referente a rankings de universidades españolas, encontramos:

- Ranking Buela-Casal (Buela-Casal et al., 2009): cuyo objetivo es analizar la productividad científica de las universidades;

- Rankings I-UGR: una serie de rankings basados en la investigación publicada en las revistas internacionales de mayor impacto (Torres-Salinas et al., 2011).

http://www.rankinguniversidades.es

Otra iniciativa que ha destacado recientemente es el proyecto U-Ranking de la Fundación BBVA y el Instituto Valenciano de Investigaciones Económicas, que presenta importantes singularidades como el hecho de permitir estudiar tres dimensiones: docencia, investigación e innovación, y desarrollo tecnológico, así como realizar dos tipos de formatos. http://www.u-ranking.es/index2.php

Finalmente, es preciso mencionar las diversas clasificaciones de las universidades españolas que realiza el Observatorio IUNE en función a una serie de indicadores de volumen de profesorado, sexenios y premios nacionales obtenidos, actividad científica, innovación, competitividad y capacidad formativa. http://www.iune.es

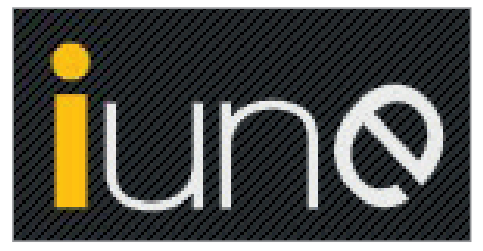

\subsection{Rankings de universidades en redes sociales}

Hasta donde alcanza nuestro conocimiento, son prácticamente inexistentes los trabajos académicos que han tratado de examinar de una manera más directa o indirecta la presencia de las universidades en las redes sociales de carácter horizontal o genéricas.

Holmberg (2015) analizó la presencia de las universidades finlandesas en varias redes sociales (Twitter, LinkedIn, YouTube, Facebook) teniendo en cuenta únicamente el número de seguidores o suscriptores. 
Otto y Williams (2014) hicieron lo propio para el caso de varias universidades africanas.

Por su parte, Permatasari et al. (2013) analizaron la penetración de las redes sociales horizontales en el sistema universitario de Indonesia, encontrando una gran variación entre instituciones de educación superior.

Campos-Freire, Rivera-Rogel y Rodríguez-Hidalgo (2014) estudiaron la presencia e impacto de las universidades andinas en Facebook, Twitter, YouTube, Google+ y ResearchGate, analizando únicamente el número de seguidores en cada una de ellas.

Paniagua-Rojano y Gómez-Calderón (2012) realizaron un ranking de las 15 universidades españolas con más seguidores en Facebook, Twitter, YouTube, LinkedIn, Tuenti y Flickr.

Al margen de estos intentos más académicos de realizar una clasificación de las universidades en RS, dada la proliferación de rankings de universidades a nivel nacional e internacional y la repercusión que tienen las RS a la hora de considerar el prestigio de las instituciones de educación superior, diversas organizaciones se han lanzado también a diseñar rankings de universidades en RS con un objetivo más centrado en obtener tráfico, notoriedad y reputación online.

En la esfera internacional podemos encontrar algunos rankings de universidades en RS, si bien sólo están centrados en universidades anglosajonas de EUA o Reino Unido. Uno de ellos es el realizado por Collegeatlas.org, que analiza la presencia e influencia social de cerca de 400 instituciones de EUA en Facebook, Twitter, Google+, LinkedIn e Instagram con más de 30 parámetros de esas redes. Con esos datos, aplicando determinados algoritmos que no quedan nada claros en cuanto a la metodología seguida, obtienen diversos rankings: Top 20 y Top 30 de influencia y presencia social en dichas redes.

http://www.collegeatlas.org/top-colleges-in-social-mediamain.html

Otro intento reciente es el realizado por The World University Rankings, que centra la atención en la presencia de universidades americanas e inglesas en Twitter. El ranking se construye de manera simple considerando el número de seguidores, Siguiendo, la ratio Seguidores/Siguiendo, la ratio tweets/día y una medida de influencia social de la cuenta obtenida con una aplicación externa de analítica de Twitter. https://www.timeshighereducation.com/news/top-100most-influential-uk-and-us-universities-on-twitter/2013373. article

Asimismo, nos encontramos con los rankings realizados por Theunipod.com para las universidades británicas en Facebook, Twitter y YouTube, si bien sólo consideran como criterio de clasificación el número de seguidores en cada una de estas redes. Además, la información es de 2013 y no ha sido actualizada.

http://www.theunipod.com/making-your-choice/ university-rankings/social-media-rankings

Al igual que ocurre en el terreno internacional, los intentos llevados a cabo para elaborar rankings de universidades españolas en RS generales son escasos. A continuación se comentan brevemente.

\section{Alianzo}

http://blogs.alianzo.com/socialnetworks

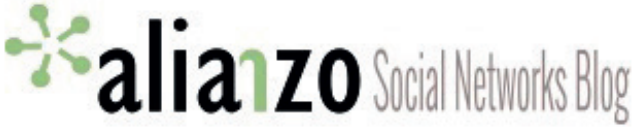

Esta institución creó inicialmente sus propios rankings, que servían para medir la popularidad de blogs por categorías basándose en los datos públicos que ofrecían dichos blogs. Conforme la empresa fue creciendo, fue haciendo rankings más relevantes incluyendo RS como Twitter y Facebook. Recientemente realizó un cambio radical en su web. En la actualidad considera una gran cantidad de RS y ofrece la posibilidad de conocer la influencia combinada de una marca en el mundo 2.0. No obstante, no se especifican los criterios de valoración y ponderación que se aplican para calcular los rankings.

\section{Avanzaentucarrera.com}

http://www.avanzaentucarrera.com/llegaraser/especiales/ especial-carreras/las-universidades-en-las-redes-sociales

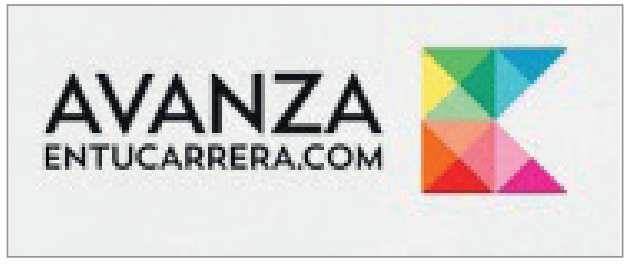

El canal de formación Infoempleo ha realizado durante dos años seguidos un ranking de universidades españolas, tanto públicas como privadas, centrándose únicamente en dos redes sociales: Facebook y Twitter. El primero de estos rankings se publicó en un blog paralelo que mantiene esta web y apenas contó con repercusión mediática. El segundo, en cambio, sí que ha producido un fuerte impacto, publicándose en múltiples medios de comunicación, la mayoría de ellos online. Este ranking se basa únicamente en el número de seguidores (presencia) que cada universidad tiene en esas RS.

\section{Blog Juan Carlos Martínez}

http://es.slideshare.net/juancarlosmtnez/informe-delestado-de-las-redes-sociales-en-las-universidades-espaolas

Es un ranking bastante completo en el que se consideran seis RS, si bien no existe transparencia en cuanto a la metodología utilizada por el autor. No se explica el procedimiento seguido ni se justifica por qué usa esos y no otros indicadores. Directamente define las fuentes de información y realiza distintos rankings. Los datos son de 2013.

\section{IY Magazine.es}

http://www.iymagazine.es/noticia/1231/universidad/top-20del-ranking-de-universidades-espanolas-en-redes-sociales. html

Ranking realizado por la web IYMagazine.es en el que el único indicador considerado es el número de fans o seguidores en las redes sociales Facebook, Twitter y Tuenti (dimensión de presencia), al igual que el ranking de Avanzaentucarrera. com. 
De la revisión realizada podemos concluir que al contrario de lo que ocurre con los rankings de universidades basados en indicadores de producción científica, los intentos por jerarquizar las universidades a través del uso de indicadores más sociales son mucho menos numerosos y en la mayoría de los casos surgen de iniciativas propias de webs o consultoras y no desde la propia academia. Esto lleva a que la metodología que se utiliza para la definición de los rankings no siempre sea del todo transparente y fiable. Además, se trata de rankings muy sesgados hacia determinados ámbitos geográficos y que se centran en muy pocas RS, en algunos casos en una sola.

\subsection{Objetivos}

El objetivo de este trabajo es proponer un marco general de definición de rankings que permita analizar el impacto de todas las universidades españolas, tanto públicas como privadas, en las principales RS genéricas (en concreto, Twitter, Facebook, Linkedln y YouTube) según un amplio número de criterios. Dicho marco se basa en:

- definición de una taxonomía de criterios que los clasifica en varias dimensiones;

- selección de los disponibles en estas RS para cada categoría;

- construcción de distintos tipos de indicadores a partir de ellos, permitiendo seleccionar la dimensión de evaluación deseada por el usuario en cada caso.

Para ello, llevaremos a cabo una recogida de datos y un establecimiento de variables lo más exhaustivo y riguroso posible.

Para ilustrar el marco de trabajo introducido, presentaremos dos casos de estudio en los que aplicaremos cuatro rankings específicos relacionados con la presencia y la influencia de las universidades españolas en Twitter y LinkedIn empleando datos recogidos en los últimos diez días de agosto de 2015. Las limitaciones de espacio no nos permiten la inclusión de resultados del resto de rankings propuestos.

\section{Metodología}

Según la Sexta oleada del Observatorio de redes sociales (The Cocktail Analysis, 2015), las tres RS horizontales más extendidas son Facebook, Twitter y Google+, por ese orden. En nuestro marco de trabajo hemos decidido seleccionar las dos primeras, dada la poca penetración de las universidades españolas en la última. Además, hemos considerado también dos RS especializadas de distinto tipo: LinkedIn como red profesional y YouTube como repositorio audiovisual. Ambas son muy empleadas por las universidades españolas como canales de comunicación activos (PaniaguaRojano; Gómez-Calderón, 2012).

Cada una de estas RS posee características distintivas y, por tanto, no es conveniente emplear los mismos indicadores en todas ellas sino hacer un diseño de rankings personalizado en cada caso. A continuación, describiremos los indicadores que se han valorado:

\section{Twitter}

- Seguidores: usuarios con un perfil personal o profesional que agregan perfiles de otros usuarios. En este caso, consideramos el número absoluto de seguidores de cada universidad.

- Ratio Siguiendo/Seguidores: proporción entre los usuarios a los que sigue una institución y los que la siguen a ella. Se trata de ver si existe un equilibrio entre ambos datos, ya que si una institución es muy seguida y por el contrario no sigue prácticamente a nadie, da lugar a una sensación de lejanía y de falta de diálogo. Cuanto más cerca de 1 esté este valor, más equilibrada será dicha proporción.

- Tweets: número de tweets generados por la cuenta de una universidad desde su creación. Mide la actividad de la universidad en Twitter. Cuanto más elevado sea, mayor será la intención de convicción por parte de la institución.

- Alcance estimado (estimated reach): alcance global estimado de los tweets de una universidad en un período de tiempo concreto, es decir, el número de cuentas únicas de Twitter que reciben los tweets.

- Exposición (exposure): número de impresiones generadas, es decir, de tweets entregados en timelines, incluidas repeticiones en un período de tiempo concreto.

\section{Facebook}

- Likes: número de usuarios que al pulsar el botón “Me gusta" se convierten en observadores activos de la web de la universidad a la que han decidido seguir.

- Internacionalización: refleja la proyección internacional que tiene cada institución. Se mide como el porcentaje de seguidores (likes) no españoles con respecto al total.

\section{LinkedIn}

- Seguidores: número de usuarios de la red social que deciden conocer cada uno de los movimientos que hace la institución dentro de la red social.

Tabla 1. Taxonomía de indicadores

\begin{tabular}{|c|c|c|c|c|}
\hline \multicolumn{5}{|c|}{ Indicadores específicos en cada red social } \\
\hline Tipo de indicador & Twitter & Facebook & LinkedIn & YouTube \\
\hline Presencia en la red & Seguidores & Likes & Seguidores & Suscriptores \\
\hline Modelo de comunicación relacional & Siguiendo/ Seguidores & -- & -- & -- \\
\hline Modelo de comunicación tradicional & Tweets & -- & -- & Vídeos \\
\hline Influencia & Exposición y Alcance estimado & -- & Interesados en asistir y Egresados & Reproducciones \\
\hline Internacionalización & -- & Internacionalización & -- & -- \\
\hline
\end{tabular}


- Empleados: número de empleados que siguen el perfil de la institución desde su perfil profesional de LinkedIn. El dato se recoge de los propios usuarios cuando especifican su puesto de trabajo y coincide con una de las instituciones presentes en la red social.

- Interesados en asistir: número de personas que han añadido la universidad a su tablón de decisiones.

- Egresados: número de antiguos alumnos que siguen a la universidad.

\section{YouTube}

- Suscriptores: número de usuarios que deciden seguir al canal de la institución para recibir notificaciones de novedades.

- Vídeos: cantidad de material multimedia que la institución ha publicado en su canal dentro de la red social.

- Reproducciones: número de veces que se ha visualizado el contenido del canal.

De este modo, podemos clasificar los indicadores seleccionados en función de su tipo, generalizando los criterios a considerar en la batería de rankings a definir, tal y como se muestra en la tabla 1.

Dado que las características de cada red social son muy distintas, no parece razonable agrupar todas las variables consideradas en un solo ranking ya que se combinarían criterios heterogéneos sin nada en común. De hecho, estos agrupamientos son complicados incluso dentro de la misma red como es el caso de Twitter o Facebook dada la diversidad de los indicadores existentes. Por esta razón, hemos optado finalmente por desvincular las distintas RS y considerarlas por separado. Al disponer de una tipología de criterios de distinta naturaleza para cada una de ellas, nuestro marco general de trabajo permite diseñar rankings específicos para medir la importancia de cada universidad en la dimensión concreta que queramos evaluar, tomando variables individuales o combinando indicadores del mismo tipo para obtener un ranking más robusto.

\section{En 2015 las redes más extendidas por nú- mero de perfiles de universidades espa- ñolas eran Facebook, Twitter y LinkedIn}

A continuación, presentamos varias propuestas de rankings para cada una de las RS consideradas en una o varias de las cinco dimensiones definidas.

\section{Twitter}

- Presencia en la red: sólo se considera la variable "Seguidores". Se trata de realizar un ranking muy básico y simultáneamente significativo pues esta variable muestra la popularidad que posee una institución en términos absolutos.

- Modelo de comunicación relacional (o de diálogo): basado en el marketing relacional donde la prioridad es la comunicación y el diálogo, es decir, que exista una complicidad y acercamiento entre emisores y receptores (Duncan; Moriarty, 1998; Bortree; Seltzer, 2009; Rybalko; Seltzer, 2010). La variable "Ratio Siguiendo/Seguidores" es la uti- lizada en este caso para conocer el grado de diálogo y conexión que existe entre los Seguidores de la universidad y la propia institución. Para definir un indicador que mida de forma adecuada la dispersión del valor obtenido en la variable con respecto a su valor óptimo de 1, proponemos emplear la siguiente expresión matemática:

$$
M C R=1-\left|1-\frac{\text { Siguiendo }}{\text { Seguidores }}\right|
$$

El indicador está basado en una medida no lineal de distancia y se define en $(0,1]$, siendo 1 su valor óptimo. AqueIlas universidades con una ratio adecuada entre Siguiendo y Seguidores toman valor 1 en el indicador mientras que las que lo superen por arriba o no lo alcancen por debajo reciben un valor que tiende a 0 cuanto más desbalanceada sea la ratio.

- Modelo de comunicación tradicional (o de persuasión): basado en el marketing tradicional en el que la emisión masiva de mensajes unidireccionales para persuadir a los grupos de interés es el único motor de comunicación (Petty; Cacioppo, 1986; 2011). Para realizar el ranking en este modelo se ha utilizado la variable "Tweets" ya que proporciona una medida directa de insistencia por parte de las instituciones.

- Indicador global de influencia en la red: pretende medir el alcance global de la influencia que tiene el perfil de la institución dentro de la red social, propuesto en el trabajo actual. Se propone combinar dos variables de la misma dimensión para construir el indicador correspondiente: "Exposición" y "Alcance estimado". Para ello se usa un operador de agregación basado en la raíz cuadrada del producto de ambas (una vez normalizadas):

\section{$I G I-T w i t t e r=\sqrt[2]{\text { Exposición } \cdot \text { Alcance estimado }}$}

Dentro de la gran variedad de operadores de agregación existentes, se ha escogido el actual porque proporciona la misma importancia a los dos criterios considerados, como pretendíamos, además de ser más restrictivo que la media al exigir valores altos en ambos para obtener un valor alto en el indicador.

\section{Facebook}

- Presencia en la red: ranking realizado con la variable "Likes", basado en datos absolutos. Es equivalente al del mismo nombre en Twitter al presentar ambos una intención de seguimiento de las instituciones en la red social.

- Internacionalización: se trata de evaluar la proyección más internacional de las universidades. Para este ranking sólo se ha considerado una variable, "Internacionalización".

\section{LinkedIn}

- Presencia en la red: al igual que en el ranking de Twitter, se considera directamente la variable "Seguidores".

- Indicador global de influencia en la red: ranking que pretende medir el alcance global de la influencia que tiene 
el perfil de la institución dentro de la red social, también propuesto por los autores del presente trabajo. Se propone combinar dos variables para construir el indicador correspondiente: "Interesados en asistir" y "Egresados". En este caso hemos decidido considerar una combinación lineal como operador de agregación ya que pensamos que las dos métricas no deberían tener el mismo grado de importancia. En nuestra opinión, los Interesados tie- nen un valor añadido al ser los que toman la iniciativa de buscar a la institución y deciden agregarla a su tablón de decisiones de LinkedIn. Por tanto, nuestro criterio fue darle un peso de 0,75 a la variable "Interesados" y de 0,25 a "Egresados" (después de normalizar ambas). El indicador toma la expresión:

IGI - LinkedIn $=0,75 \cdot$ Interesados $+0,25 \cdot$ Egresados

Tabla 2. Cuentas oficiales de universidades españolas en Twitter consideradas en el presente estudio:

\begin{tabular}{|c|c|c|}
\hline & Universidad & Username \\
\hline IEU & IE Universidad & @leuniversity \\
\hline UMU & Mondragón Unibersitatea & @Munibertsitatea \\
\hline UDIMA & Universidad a Distancia de Madrid & @UDIMA \\
\hline UAX & Universidad Alfonso X El Sabio & @universidad_uax \\
\hline UAN & Universidad Antonio de Nebrija & @Nebrija \\
\hline UAM & Universidad Autónoma de Madrid & @UAM_Madrid \\
\hline UCJC & Universidad Camilo José Cela & @universidadcjc \\
\hline UCH-CEU & Universidad Cardenal Herrera-CEU & @uchceu \\
\hline UC3M & Universidad Carlos III de Madrid & @uc3m \\
\hline UCV & $\begin{array}{l}\text { Universidad Católica de Valencia San } \\
\text { Vicente Mártir }\end{array}$ & @UCV_es \\
\hline UCAM & Universidad Católica San Antonio & @UCAM \\
\hline UCST & $\begin{array}{l}\text { Universidad Católica Santa Teresa de } \\
\text { Jesús de Avila }\end{array}$ & @UCAVILA \\
\hline UCM & Universidad Complutense de Madrid & @unicomplutense \\
\hline UAH & Universidad de Alcalá de Henares & @UAHes \\
\hline$U A$ & Universidad de Alicante & @UA_Universidad \\
\hline$U A L$ & Universidad de Almería & @ualmeria \\
\hline UBU & Universidad de Burgos & @UBUEstudiantes \\
\hline$U C A$ & Universidad de Cádiz & @univcadiz \\
\hline UNICAN & Universidad de Cantabria & @unican \\
\hline$U C L M$ & Universidad de Castilla-La Mancha & @uclm_es \\
\hline UCO & Universidad de Córdoba & @Univcordoba \\
\hline UDE & Universidad de Deusto & @deusto \\
\hline UEX & Universidad de Extremadura & @infouex \\
\hline UGR & Universidad de Granada & @CanalUGR \\
\hline$U H U$ & Universidad de Huelva & @CanalUHU \\
\hline UJA & Universidad de Jaén & @ujaen \\
\hline$U L L$ & Universidad de La Laguna & @CanalULL \\
\hline UR & Universidad de La Rioja & @Unirioja \\
\hline ULPGC & $\begin{array}{l}\text { Universidad de Las Palmas de Gran } \\
\text { Canaria }\end{array}$ & @ULPGC \\
\hline ULE & Universidad de León & @unileon \\
\hline UMA & Universidad de Málaga & $@$ @InfoUMA \\
\hline$U M$ & Universidad de Murcia & @umnoticias \\
\hline UNA & Universidad de Navarra & @unav \\
\hline Uniovi & Universidad de Oviedo & @uniovi_info \\
\hline USAL & Universidad de Salamanca & @usal \\
\hline USC & Universidad de Santiago de Compostela & @UniversidadeUSC \\
\hline USE & Universidad de Sevilla & @unisevilla \\
\hline UVA & Universidad de Valladolid & @UVa_es \\
\hline UVIGO & Universidad de Vigo & @uvigo \\
\hline
\end{tabular}

\begin{tabular}{|c|c|c|}
\hline UZ & Universidad de Zaragoza & @unizar \\
\hline UPV-EHU & $\begin{array}{l}\text { Universidad del País Vasco/Euskal } \\
\text { Herriko Unibertsitatea }\end{array}$ & @upvehu \\
\hline UEM & Universidad Europea de Madrid & @UEuropea \\
\hline UEMC & $\begin{array}{l}\text { Universidad Europea Miguel de } \\
\text { Cervantes }\end{array}$ & @UEMC \\
\hline UFV & Universidad Francisco de Vitoria & @ufvmadrid \\
\hline UNIA & Universidad Internacional de Andalucía & @UNIAuniversidad \\
\hline UIC & Universidad Internacional de Catalunya & @UICbarcelona \\
\hline UNIR & Universidad Internacional de La Rioja & @UNIRuniversidad \\
\hline UIIC & $\begin{array}{l}\text { Universidad Internacional Isabel I de } \\
\text { Castilla }\end{array}$ & @ui1Universidad \\
\hline UIMP & $\begin{array}{l}\text { Universidad Internacional Menéndez } \\
\text { Pelayo }\end{array}$ & @UIMP \\
\hline UIV & Universidad Internacional Valenciana & @UniversidadVIU \\
\hline ונU & Universidad Jaume I de Castellón & @UJI_noticies \\
\hline ULA & Universidad Loyola Andalucía & @LoyolaAnd \\
\hline UMH & Universidad Miguel Hernández de Elche & @UniversidadMH \\
\hline UNED & $\begin{array}{l}\text { Universidad Nacional de Educación a } \\
\text { Distancia }\end{array}$ & @UNED \\
\hline UPO & Universidad Pablo de Olavide & @pablodeolavide \\
\hline UPCA & Universidad Politécnica de Cartagena & @UPCTnoticias \\
\hline UPM & Universidad Politécnica de Madrid & @La_UPM \\
\hline UPCO & Universidad Pontificia de Comillas & @UCOMILLAS \\
\hline UPSAL & Universidad Pontificia de Salamanca & @upsa_salamanca \\
\hline UPN & Universidad Pública de Navarra & @Unavarra \\
\hline URJC & Universidad Rey Juan Carlos & @urjc \\
\hline USJ & Universidad San Jorge & @_usj_ \\
\hline USP-CEU & Universidad San Pablo CEU & @USPCEU \\
\hline UDC & Universidade da Coruña & @UDC_gal \\
\hline UAO-CEU & Universitat Abat Oliba CEU & @UAOCEU \\
\hline$U A B$ & Universitat Autònoma de Barcelona & @UAB_info \\
\hline UB & Universitat de Barcelona & @UB_endirecte \\
\hline UGI & Universitat de Girona & @univgirona \\
\hline UIB & Universitat de les Illes Balears & @UIBuniversitat \\
\hline$U L$ & Universitat de Lleida & @trampoli_udl \\
\hline UV & Universitat de València-Estudi General & $@ U V \_E G$ \\
\hline UVIC & Universitat de Vic & @uvic_news \\
\hline UOC & Universitat Oberta de Catalunya & @UOCuniversitat \\
\hline UPC & Universitat Politècnica de Catalunya & @BarcelonaTech \\
\hline UPV & Universitat Politècnica de València & @upv \\
\hline UPF & Universitat Pompeu Fabra & @univpompeufabra \\
\hline URL & Universitat Ramon Llull & @uramonllull \\
\hline URV & Universitat Rovira i Virgili & @universitatURV \\
\hline
\end{tabular}




\section{YouTube}

- Presencia en la red: se considera únicamente la variable "Suscriptores", que representa el mismo concepto que las consideradas en las RS anteriores para esta dimensión.

- Modelo de comunicación tradicional: se utiliza la variable "Vídeos" como medida directa de insistencia por parte de las instituciones.

- Influencia: Se emplea la variable "Reproducciones" para este ranking.

\section{Resultados}

Con objeto de ilustrar la metodología presentada, vamos a evaluar los resultados de las universidades españolas en algunos de nuestros rankings. En concreto, hemos seleccionado dos RS de distinta naturaleza: Twitter (una red horizontal masiva) y Linkedln (una red vertical dado su perfil más profesional y orientado); y dos rankings específicos que miden las mismas dimensiones para cada una de ellas, Presencia e Influencia.

Se ha trabajado con prácticamente toda la población de universidades españolas (78), tanto públicas como privadas (tabla 2). Tal y como se ha indicado a lo largo de este trabajo, nos pareció importante que se consideraran relevantes todas las universidades españolas en igualdad de condiciones para la definición de unos rankings verdaderamente útiles. Sólo se ha dejado fuera la Universidad Europea del Atlántico ya que, dada su reciente creación, presenta todavía una escasa presencia en RS.

El estudio se ha basado en la recogida de datos de varias fuentes. Los datos más significativos y numerosos se han extraído de las propias RS, aunque para Twitter se han empleado también las dos métricas que definen el ranking de influencia obtenidas de Tweetreach.com. Todas las cuentas de las dos RS consideradas en este estudio son las oficiales de las propias universidades recogidas en sus webs oficiales. En el caso de Linkedln la cuenta de cada universidad se corresponde con el propio nombre de la misma. En el caso de Twitter, el username de cada universidad está incluido en la tabla 2.

La recogida de información se realizó entre los días 20 y 30 de agosto de 2015. Uno de los handicaps más importantes fue la falta de normalización del nombre de las universidades en cada una de las RS, lo que requirió un importante trabajo adicional.

Una vez establecidas las variables con toda su información, se procedió a la realización de los cálculos pertinentes para obtener los valores de los indicadores y a la ordenación de las instituciones con respecto a dichos valores en cada ranking. Además, se analizó la clasificación de las universidades en cuatro de las combinaciones por pares de los rankings definidos para proporcionar una visión más general de su posicionamiento.

\subsection{Rankings de universidades en Twitter}

\subsubsection{Ranking de Presencia en Twitter}

Los resultados de este primer ranking están recogidos en la tabla 3. Según el indicador de presencia en Twitter, la Universidad de Granada estaría a la cabeza con casi 90.000 se- guidores. El segundo y tercer lugar lo ocuparían otras dos universidades públicas, UNED y Sevilla, respectivamente. Además, podemos ver que el top ten de las universidades de esta clasificación es de carácter público, salvo por la presencia de la Universidad Europea de Madrid.

\subsubsection{Ranking de Influencia global en Twitter}

El segundo de los rankings definidos sobre Twitter está relacionado con el grado de Influencia global en la red

Tabla 3. Ranking de Presencia en Twitter

\begin{tabular}{|c|c|c|c|c|c|}
\hline $\begin{array}{l}\text { Posi- } \\
\text { ción }\end{array}$ & Univ. & Seguidores & Posición & Univ. & Seguidores \\
\hline 1 & $U G R$ & 89.471 & 40 & $U M H$ & 14.252 \\
\hline 2 & UNED & 81.381 & 41 & UCAM & 14.218 \\
\hline 3 & US & 79.582 & 42 & UIMP & 13.046 \\
\hline 4 & UMA & 59.642 & 43 & $U Z$ & 12.892 \\
\hline 5 & $U M$ & 51.054 & 44 & UIC & 12.871 \\
\hline 6 & UCM & 43.701 & 45 & UCJC & 11.449 \\
\hline 7 & $U A$ & 43.299 & 46 & $U R$ & 11.275 \\
\hline 8 & UV & 37.640 & 47 & UPN & 11.159 \\
\hline 9 & UAM & 35.512 & 48 & UAN & 10.523 \\
\hline 10 & UEM & 34.540 & 49 & UNIR & 10.045 \\
\hline 11 & URJC & 32.615 & 50 & ال & 9.658 \\
\hline 12 & UJ & 32.604 & 51 & UNICAN & 9.202 \\
\hline 13 & USAL & 27.520 & 52 & $U I B$ & 8.939 \\
\hline 14 & $U A H$ & 26.886 & 53 & URV & 8.592 \\
\hline 15 & UPV & 26.596 & 54 & UCST & 8.111 \\
\hline 16 & $U C A$ & 26.363 & 55 & $U B U$ & 7.792 \\
\hline 17 & UVA & 26.259 & 56 & $U C H-C E U$ & 7.663 \\
\hline 18 & UNA & 25.995 & 57 & UNIA & 7.404 \\
\hline 19 & UOC & 25.860 & 58 & UDIMA & 6.960 \\
\hline 20 & UCO & 25.844 & 59 & UFV & 6.937 \\
\hline 21 & ULPGC & 25.815 & 60 & USP-CEU & 6.758 \\
\hline 22 & $U B$ & 25.777 & 61 & UVIC & 6.644 \\
\hline 23 & $U L L$ & 24.102 & 62 & IEU & 6.467 \\
\hline 24 & UPM & 23.724 & 63 & UCV & 6.390 \\
\hline 25 & UVIGO & 22.607 & 64 & UNIOVI & 6.259 \\
\hline 26 & UC3M & 22.197 & 65 & $U R L$ & 5.988 \\
\hline 27 & UGI & 21.467 & 66 & $U M U$ & 5.606 \\
\hline 28 & UPO & 21.149 & 67 & ULA & 5.223 \\
\hline 29 & $U A B$ & 21.084 & 68 & UEMC & 4.997 \\
\hline 30 & $U D C$ & 20.464 & 69 & UAX & 4.871 \\
\hline 31 & UCLM & 20.285 & 70 & UIV & 4.659 \\
\hline 32 & $U H U$ & 19.294 & 71 & UPCO & 4.484 \\
\hline 33 & $U A L$ & 18.646 & 72 & USC & 4.143 \\
\hline 34 & $U P C$ & 17.518 & 73 & UAO-CEU & 3.380 \\
\hline 35 & $U D E$ & 17.380 & 74 & UPCA & 3.037 \\
\hline 36 & ULE & 17.242 & 75 & UIIC & 2.684 \\
\hline 37 & UPF & 17.052 & 76 & UPSAL & 2.065 \\
\hline 38 & UEX & 14.710 & 77 & USJ & 1.538 \\
\hline 39 & UPV-EHU & 14.388 & 78 & ULL & 680 \\
\hline
\end{tabular}


$(I G R)$ que tienen las universidades en esta red social (tabla 4). Recordamos que este ranking se define a partir de la combinación de los indicadores "Exposición" y "Alcance estimado". En esta ocasión es la UC3M la que ocupa el primer lugar, seguida muy de cerca la UIIC y la UFV. Merece la pena destacar que existe cierto equilibrio entre las universidades públicas y privadas en el top ten: seis públicas y cuatro privadas. Dado el menor número de universidades privadas y comparando con los resultados del resto de rankings presentados, parece ser que este tipo de universidades realizan una apuesta por un modelo de comunicación basado en esta red social.

\subsection{Rankings de universidades en LinkedIn}

\subsubsection{Ranking de Presencia en LinkedIn}

La UCM es la que lidera la clasificación de forma destacada con casi 250.000 seguidores, seguida de la UB y la UPM (tabla 5). Cabe destacar que las diez primeras posiciones de esta clasificación están ocupadas únicamente por universidades públicas.
Tabla 4. Ranking de Influencia global en Twitter

\begin{tabular}{|c|c|c|c|c|c|}
\hline $\begin{array}{l}\text { Posi- } \\
\text { ción }\end{array}$ & Univ. & IGR & Posición & Univ. & IGR \\
\hline 1 & UC3M & 0,9879 & 40 & UPSAL & 0,2813 \\
\hline 2 & UIIC & 0,9642 & 41 & $U M U$ & 0,2811 \\
\hline 3 & UFV & 0,8227 & 42 & UV & 0,2779 \\
\hline 4 & USE & 0,7424 & 43 & UCH-CEU & 0,2620 \\
\hline 5 & $U D C$ & 0,7390 & 44 & $U H U$ & 0,2611 \\
\hline 6 & UCLM & 0,5700 & 45 & USP-CEU & 0,2607 \\
\hline 7 & UIMP & 0,5448 & 46 & UAO-CEU & 0,2599 \\
\hline 8 & $U M$ & 0,5291 & 47 & UPF & 0,2521 \\
\hline 9 & $U P C$ & 0,4968 & 48 & UNIA & 0,2418 \\
\hline 10 & $U D E$ & 0,4816 & 49 & ال & 0,2413 \\
\hline 11 & UPN & 0,4728 & 50 & IEU & 0,2407 \\
\hline 12 & UNA & 0,4560 & 51 & UCV & 0,2394 \\
\hline 13 & $U B$ & 0,4558 & 52 & UGI & 0,2394 \\
\hline 14 & UNIR & 0,4518 & 53 & UCJC & 0,2346 \\
\hline 15 & UAM & 0,4471 & 54 & UNICAN & 0,2245 \\
\hline 16 & UPV & 0,4336 & 55 & $U A H$ & 0,2211 \\
\hline 17 & UEM & 0,4330 & 56 & ULE & 0,2146 \\
\hline 18 & URV & 0,4290 & 57 & UPV-EHU & 0,2144 \\
\hline 19 & $U R L$ & 0,4279 & 58 & UNED & 0,2121 \\
\hline 20 & UGR & 0,4204 & 59 & UEX & 0,2032 \\
\hline 21 & USAL & 0,4181 & 60 & UMA & 0,2018 \\
\hline 22 & UCM & 0,3702 & 61 & $U A L$ & 0,1939 \\
\hline 23 & UPCA & 0,3682 & 62 & USC & 0,1828 \\
\hline 24 & URJC & 0,3674 & 63 & $U B U$ & 0,1673 \\
\hline 25 & $U Z$ & 0,3551 & 64 & ULA & 0,1664 \\
\hline 26 & UJA & 0,3539 & 65 & UDIMA & 0,1637 \\
\hline 27 & ULPGC & 0,3454 & 66 & UCAM & 0,1625 \\
\hline 28 & UPM & 0,3437 & 67 & UEMC & 0,1518 \\
\hline 29 & $U A B$ & 0,3434 & 68 & $U L L$ & 0,1415 \\
\hline 30 & UR & 0,3363 & 69 & $U A$ & 0,1392 \\
\hline 31 & UIC & 0,3270 & 70 & UNIOVI & 0,1385 \\
\hline 32 & $U C A$ & 0,3139 & 71 & UVIGO & 0,1365 \\
\hline 33 & UPCO & 0,3046 & 72 & $U A X$ & 0,1337 \\
\hline 34 & $U I B$ & 0,3039 & 73 & UPO & 0,1201 \\
\hline 35 & UVIC & 0,3035 & 74 & $U M H$ & 0,1119 \\
\hline 36 & UVA & 0,3010 & 75 & USJ & 0,1030 \\
\hline 37 & UOC & 0,2871 & 76 & UIV & 0,0907 \\
\hline 38 & UCST & 0,2858 & 77 & UAN & 0,0732 \\
\hline 39 & UCO & 0,2821 & 78 & $U L$ & 0,0145 \\
\hline
\end{tabular}

Tabla 5. Ranking de Presencia en Linkedln

\begin{tabular}{|c|c|c|c|c|c|}
\hline $\begin{array}{l}\text { Posi- } \\
\text { ción }\end{array}$ & Univ & Seguidores & $\begin{array}{l}\text { Posi- } \\
\text { ción }\end{array}$ & Univ & Seguidores \\
\hline 1 & UCM & 249.297 & 40 & URV & 18.082 \\
\hline 2 & $U B$ & 164.182 & 41 & UNICAN & 17.879 \\
\hline 3 & UPM & 116.901 & 42 & UPO & 17.801 \\
\hline 4 & $U A B$ & 93.198 & 43 & UPSAL & 17.399 \\
\hline 5 & USE & 91.316 & 44 & ULE & 17.334 \\
\hline 6 & $U P C$ & 86.424 & 45 & UCO & 17.207 \\
\hline 7 & UAM & 78.508 & 46 & $U C A$ & 16.847 \\
\hline 8 & UGR & 74.702 & 47 & UAN & 16.516 \\
\hline 9 & UPV & 62.219 & 48 & UCJC & 15.916 \\
\hline 10 & USAL & 58.722 & 49 & $U A X$ & 15.301 \\
\hline 11 & IEU & 55.203 & 50 & $U M H$ & 15.016 \\
\hline 12 & $U P F$ & 54.675 & 51 & $U I B$ & 13.082 \\
\hline 13 & UV & 54.257 & 52 & UFV & 12.648 \\
\hline 14 & UC3M & 54.211 & 53 & UJA & 12.527 \\
\hline 15 & UOC & 53.009 & 54 & $U H U$ & 11.829 \\
\hline 16 & UNED & 51.399 & 55 & UPN & 11.292 \\
\hline 17 & $U Z$ & 49.924 & 56 & $U L$ & 11.251 \\
\hline 18 & URJC & 44.492 & 57 & $U A L$ & 11.004 \\
\hline 19 & UPV-EHU & 43.467 & 58 & $U B U$ & 10.529 \\
\hline 20 & USC & 42.985 & 59 & UIC & 8.570 \\
\hline 21 & $U A$ & 41.770 & 60 & $U R$ & 8.274 \\
\hline 22 & UNIOVI & 40.308 & 61 & UVIC & 7.779 \\
\hline 23 & UVA & 40.291 & 62 & $U P C A$ & 7.716 \\
\hline 24 & $U A H$ & 39.296 & 63 & UCAM & 7.682 \\
\hline 25 & UMA & 38.988 & 64 & UMU & 7.597 \\
\hline 26 & USP-CEU & 36.305 & 65 & UCV & 6.668 \\
\hline 27 & UNA & 35.816 & 66 & UEMC & 6.029 \\
\hline 28 & $U D E$ & 35.531 & 67 & $U A O-C E U$ & 5.294 \\
\hline 29 & UPCO & 33.115 & 68 & UIMP & 4.458 \\
\hline 30 & $U M$ & 31.659 & 69 & UNIR & 4.360 \\
\hline 31 & UVIGO & 30.584 & 70 & USJ & 3.636 \\
\hline 32 & UEM & 30.093 & 71 & UNIA & 2.828 \\
\hline 33 & URL & 27.359 & 72 & UDIMA & 2.760 \\
\hline 34 & UCLM & 25.866 & 73 & ال & 2.503 \\
\hline 35 & UEX & 22.938 & 74 & UCST & 2.446 \\
\hline 36 & ULL & 21.895 & 75 & $U C H-C E U$ & 1.103 \\
\hline 37 & UDC & 21.110 & 76 & ULA & 966 \\
\hline 38 & ULPGC & 20.320 & 77 & UIV & 740 \\
\hline 39 & UGI & 19.329 & 78 & UIIC & 422 \\
\hline
\end{tabular}




\subsubsection{Ranking de Influencia global en LinkedIn}

En la clasificación de Influencia global, en la que consideraban las variables "Interesados en asistir" y "Egresados", la IEU alcanza el primer puesto con un valor de 0,7992 (tabla 6). Le siguen a corta distancia la UCM y la UB. De nuevo, como en el ranking anterior, predominan las universidades públicas en el top ten, siendo la única de carácter privado la que ocupa el primer lugar.

Tabla 6. Ranking de Influencia global en Linkedln

\begin{tabular}{|c|c|c|c|c|c|}
\hline $\begin{array}{l}\text { Posi- } \\
\text { ción }\end{array}$ & Univ & Influencia & $\begin{array}{l}\text { Posi- } \\
\text { ción }\end{array}$ & Univ & Influencia \\
\hline 1 & IEU & 0,7992 & 40 & UNIR & 0,0647 \\
\hline 2 & UCM & 0,7762 & 41 & UCJC & 0,0641 \\
\hline 3 & $U B$ & 0,6542 & 42 & ULPGC & 0,0640 \\
\hline 4 & $U A B$ & 0,4425 & 43 & URV & 0,0639 \\
\hline 5 & UAM & 0,4287 & 44 & UPO & 0,0636 \\
\hline 6 & UPM & 0,3965 & 45 & $U I B$ & 0,0565 \\
\hline 7 & UGR & 0,3419 & 46 & ULE & 0,0556 \\
\hline 8 & UPF & 0,3371 & 47 & UAN & 0,0544 \\
\hline 9 & UPC & 0,3201 & 48 & UPSAL & 0,0529 \\
\hline 10 & USE & 0,3175 & 49 & UFV & 0,0528 \\
\hline 11 & UOC & 0,2958 & 50 & UCO & 0,0528 \\
\hline 12 & UV & 0,2871 & 51 & UNICAN & 0,0525 \\
\hline 13 & UNED & 0,2747 & 52 & UJA & 0,0520 \\
\hline 14 & UPV & 0,2726 & 53 & UCAM & 0,0493 \\
\hline 15 & USAL & 0,2577 & 54 & UEX & 0,0482 \\
\hline 16 & UC3M & 0,2478 & 55 & $U M H$ & 0,0468 \\
\hline 17 & URJC & 0,1785 & 56 & ונU & 0,0460 \\
\hline 18 & $U A H$ & 0,1771 & 57 & $U A X$ & 0,0438 \\
\hline 19 & UEM & 0,1628 & 58 & UEMC & 0,0437 \\
\hline 20 & UPV-EHU & 0,1618 & 59 & UMU & 0,0436 \\
\hline 21 & UMA & 0,1535 & 60 & $U A L$ & 0,0417 \\
\hline 22 & $U A$ & 0,1496 & 61 & $U C H-C E U$ & 0,0399 \\
\hline 23 & USC & 0,1471 & 62 & UCV & 0,0394 \\
\hline 24 & UPCO & 0,1401 & 63 & UVIC & 0,0391 \\
\hline 25 & UNA & 0,1392 & 64 & UPN & 0,0386 \\
\hline 26 & $U Z$ & 0,1369 & 65 & UIC & 0,0372 \\
\hline 27 & $U D E$ & 0,1295 & 66 & $U B U$ & 0,0334 \\
\hline 28 & UVA & 0,1080 & 67 & $U H U$ & 0,0300 \\
\hline 29 & UNIOVI & 0,1069 & 68 & $U L$ & 0,0295 \\
\hline 30 & $U M$ & 0,0933 & 69 & $U R$ & 0,0294 \\
\hline 31 & USP-CEU & 0,0910 & 70 & UNIA & 0,0241 \\
\hline 32 & UDIMA & 0,0909 & 71 & $U P C A$ & 0,0241 \\
\hline 33 & UVIGO & 0,0866 & 72 & ULA & 0,0239 \\
\hline 34 & UCLM & 0,0745 & 73 & UIV & 0,0217 \\
\hline 35 & $U D C$ & 0,0732 & 74 & $U A B-C E U$ & 0,0203 \\
\hline 36 & ULL & 0,0716 & 75 & UIMP & 0,0186 \\
\hline 37 & URL & 0,0692 & 76 & USJ & 0,0174 \\
\hline 38 & UGI & 0,0652 & 77 & UCST & 0,0148 \\
\hline 39 & $U C A$ & 0,0652 & 78 & UIIC & 0,0007 \\
\hline
\end{tabular}

\subsection{Estudio conjunto de los resultados de los rankings}

En esta sección pretendemos profundizar aún más en el posicionamiento de las universidades analizando algunos resultados combinados de los rankings que hemos definido. Para ello vamos a realizar unos gráficos de dispersión que representan la posición conjunta de cada universidad en dos rankings distintos. Por un lado, analizaremos conjuntamente los dos rankings definidos para cada red social mientras que por otro relacionaremos las dos dimensiones analizadas, presencia e influencia, en ambas RS. En los cuatro casos, realizaremos un análisis estadístico para determinar el grado de correlación lineal existente entre los dos indicadores considerados, empleando para ello el coeficiente $r$ de Pearson.

\subsubsection{Estudio conjunto de los rankings de Presencia e In- fluencia en Twitter}

En este primer caso, el valor del coeficiente $r$ obtenido es de 0,198 , lo que indica que existe una correlación muy poco significativa entre los dos indicadores considerados. De este modo, queda claro el hecho de que una presencia alta en Twitter no implica directamente una influencia importante en esta RS y viceversa.

Como se observa en la figura 1, la Universidad de Sevilla es la que tiene un equilibrio más destacable entre influencia y presencia en Twitter, siendo la tercera en presencia y la cuarta en influencia. Vemos que la primera y la segunda en presencia, Granada y la UNED, ocupan posiciones muy lejanas a la primera en Influencia, la Universidad Carlos III de Madrid. Esto puede deberse a que, a pesar de tener una fuerte actividad en la red y un gran volumen de seguidores, el alcance obtenido no se corresponde con su posición en términos de presencia.

La razón de ello puede estar en la "calidad" de los seguidores de esas universidades dado que la influencia depende del número de seguidores que tienen los seguidores de las universidades y de la actividad que estos ejerzan en la red social. Isabel I de Castilla y Francisco de Vitoria son universidades que, a pesar de tener una presencia muy escasa en Twitter, presentan una potente influencia ocupando el segundo y tercer lugar respectivamente. El caso de la propia Carlos III y la de A Coruña es similar, aunque en este caso la presencia es bastante más destacada.

\subsubsection{Estudio conjunto de los rankings de Presencia e In- fluencia en LinkedIn}

Las dos variables presentan una correlación muy significativa en la que apenas se aprecian elementos demasiado desmarcados (figura 2). El valor del coeficiente $r$ de Pearson obtenido en el análisis realizado es de 0,877 , señalando una correlación positiva bastante alta. Podemos concluir, por tanto, que al contrario que en el caso de Twitter, en la red social LinkedIn la presencia y la influencia de las universidades españolas en la red están fuertemente relacionadas.

La Universidad Complutense de Madrid y la Universitat de Barcelona son dos claros ejemplos de equilibrio entre presencia e influencia en LinkedIn. Ambas universidades cuentan con un nutrido número de seguidores y además son instituciones consideradas muy positivamente por éstos. Cabe 


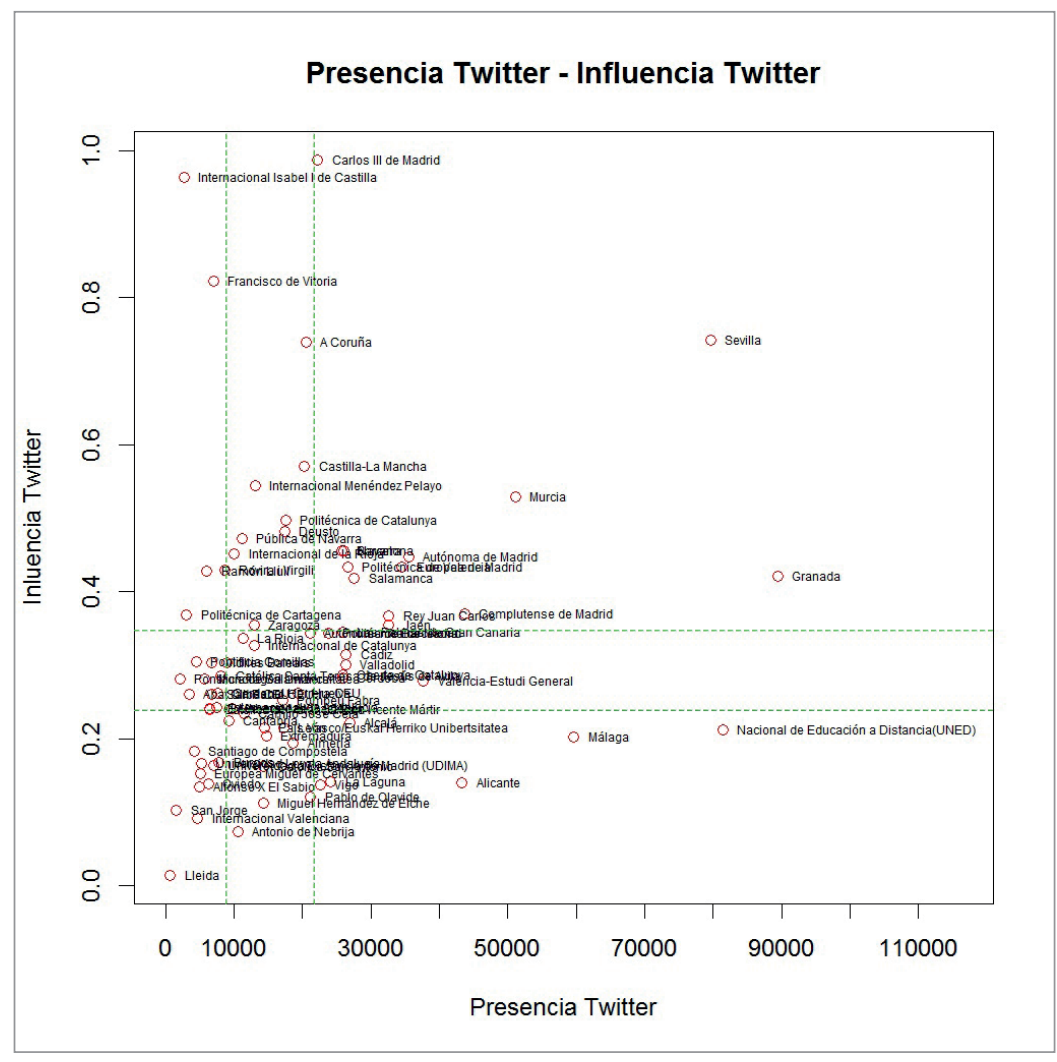

Figura 1. Gráfico de dispersión de los rankings de presencia e influencia en Twitter

\subsubsection{Estudio conjunto de los rankings de Influencia en Twitter y LinkedIn}

En este caso no se aprecia una correlación significativa entre las influencias de las RS Twitter y LinkedIn (coeficiente $r$ : 0,167). EI grueso de las universidades que destacan en una de las dos, tiene una influencia inferior al 0,5 en la otra. Este hecho puede deberse a una posible focalización del esfuerzo de comunicación en una sola de estas dos RS por parte de las universidades españolas.

En los extremos se sitúan por un lado IE Universidad con una fuerte influencia en Linkedln, la más importante, y una muy baja en Twitter. Por otro lado, la Internacional Isabel I de Castilla, situada en segunda posición en cuanto a influencia en Twitter y en cambio prácticamente inexistente en LinkedIn. En lo que respecta a universidades con un mejor equilibrio, podemos destacar a la Carlos III, primera en influencia en Twitter y decimosexta en LinkedIn; a la de Sevilla, cuarta en Twitter y décima en LinkedIn; a la de Barcelona, tercera en LinkedIn y decimotercera en Twitter; a la Autónoma de Madrid, quinta en Linkedln y

destacar el caso de la IE Universidad que cuenta con una importante influencia en LinkedIn con una presencia poco relevante. Esto puede deberse a que es una universidad que tiene mucho prestigio y que, por tanto, cuenta con un alto número de personas que tienen la intención de asistir. En cambio, su actividad en la red es básicamente testimonial.

\subsubsection{Estudio conjunto de los rankings de Presencia en Twitter y LinkedIn}

En este caso, el coeficiente de Pearson toma un valor de 0,47 , lo que muestra una correlación positiva bastante moderada. Se puede concluir que la apuesta por la presencia conjunta de las universidades españolas en estas dos RS no es excesivamente importante.

La Complutense de Madrid es la que tiene la presencia combinada más destacable, siendo la primera en Linkedln y la sexta en Twitter (véase la figura 3). La primera en Twitter, Granada, es la octava en LinkedIn, presentando también un buen equilibrio, al igual que Sevilla (tercera en Twitter y quinta en LinkedIn). El resto de universidades destacadas en alguna de las dos RS, no se encuentran en una posición tan señalada en la otra, como es el caso de Barcelona, Politécnica de Madrid, Autònoma de Barcelona, Politècnica de Catalunya (con mayor presencia en LinkedIn) y UNED, Málaga, Murcia y Alicante (más destacadas en Twitter).

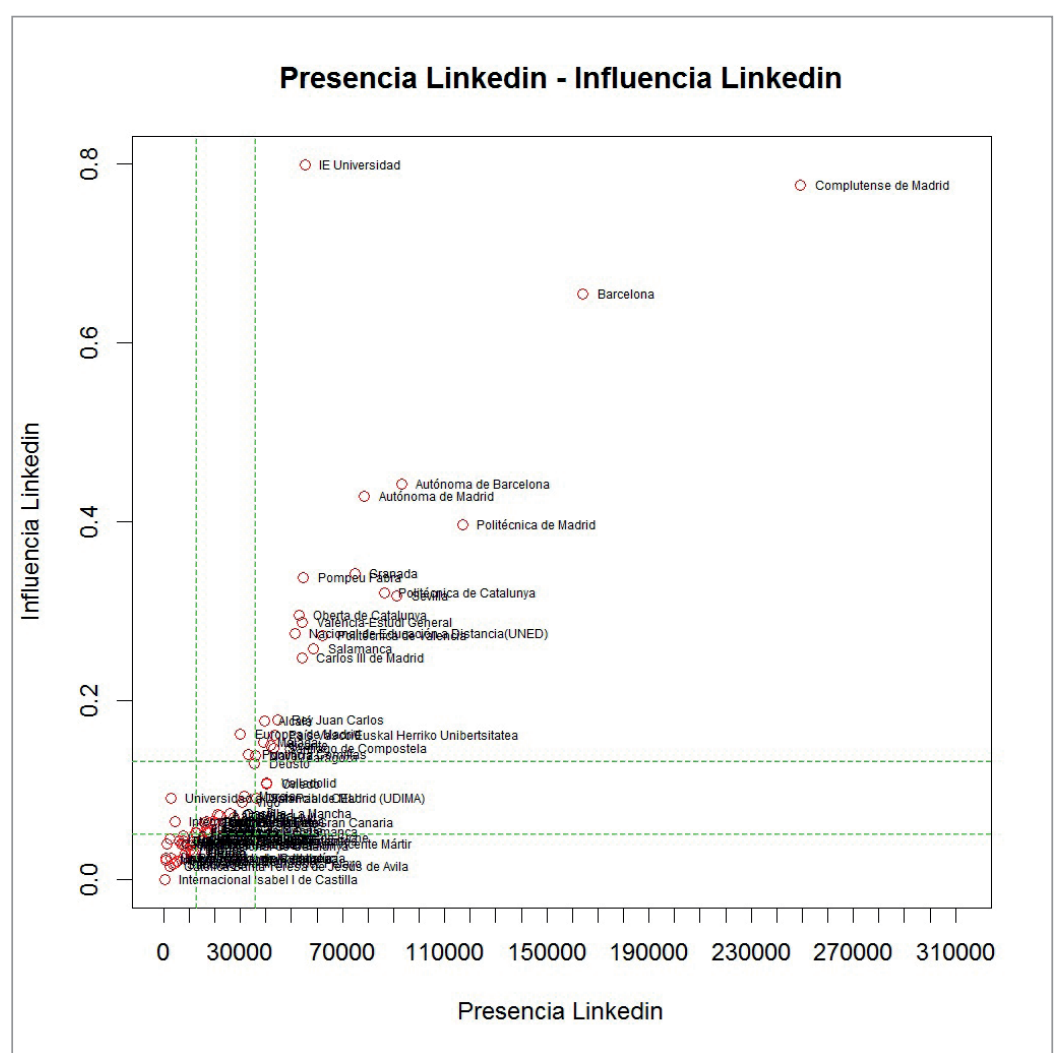

Figura 2. Gráfico de dispersión de los rankings de presencia e influencia en LinkedIn 


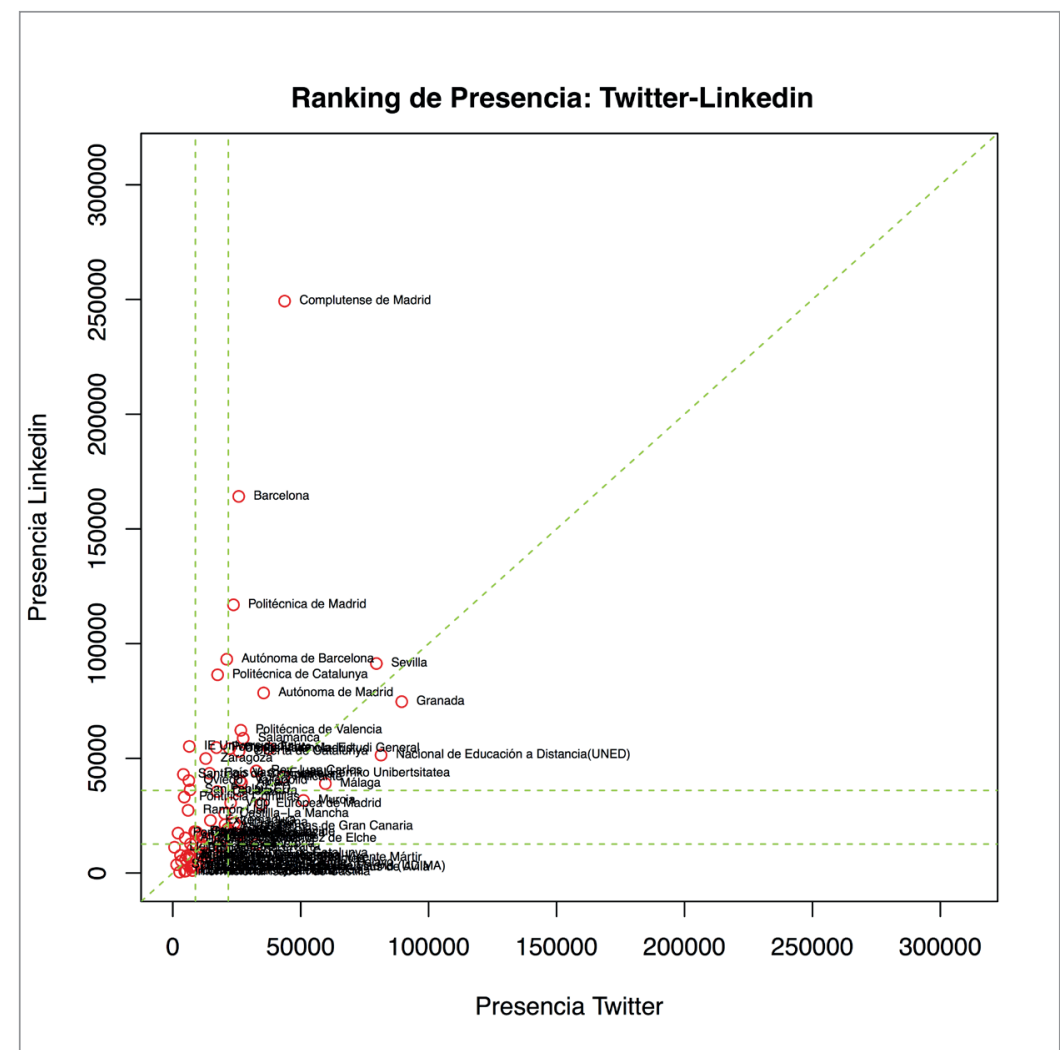

Figura 3. Gráfico de dispersión de los rankings de presencia en Twitter y Linkedln das las universidades españolas en todos los rankings realizados (siempre que, por supuesto, tengan presencia y existan datos de las mismas para la red social concreta), se explicitan claramente los indicadores empleados en cada caso, seleccionando aquellos más relevantes para el propósito de cada ranking de acuerdo con la naturaleza específica de la red social considerada, se señalan los mecanismos de agregación considerados para combinar los indicadores para la creación de cada ranking, y se realiza un análisis exhaustivo de los resultados obtenidos.

Del análisis de las cuentas institucionales de las universidades españolas a fecha del trabajo de campo (agosto de 2015), podemos concluir que las redes más extendidas por número de perfiles de universidades españolas en ese momento eran Facebook, Twitter y LinkedIn (todas ellas con 78 cuentas, resultando en un 93,9\% de penetración), seguidas por YouTube (74 cuentas, $89,1 \%)$. Los indicadores seleccionados y clasificados en dimensiones de diferente naturaleza permiten diseñar una amplia batería de rankings para las cuatro RS consideradas. Se pueden proponer directa-

\section{Conclusiones y líneas futuras de trabajo}

En este trabajo hemos propuesto un marco general para la definición de rankings personalizados de medición del impacto de las universidades españolas en redes sociales (RS) con respecto a varias dimensiones. Usando este marco hemos construido rankings a partir de conjuntos de datos relevantes que permiten entender el funcionamiento de las universidades en RS a un nivel desconocido hasta ahora. La metodología seguida ha sido rigurosa y está alineada con las características que deben tener los rankings óptimos según la bibliografía (Merisotis, 2002).

Las propuestas presentadas superan las limitaciones de otros intentos realizados hasta la fecha que manifestaban notables carencias como la especificidad, al usar un número bajo de redes sociales (Avanzaentucarrera.com); la falta de cobertura al considerar un número limitado de universidades (varios de los rankings existentes); la falta de transparencia en la metodología de definición de los rankings (Alianzo, Blog Juan Carlos Martínez), e incluso la definición de indicadores y la realización de una interpretación de datos excesivamente simples (Avanzaentucarrera.com, IY Magazine.es). En nuestro marco de trabajo se propone una metodología para cuatro de las RS más extendidas, se consideran to-

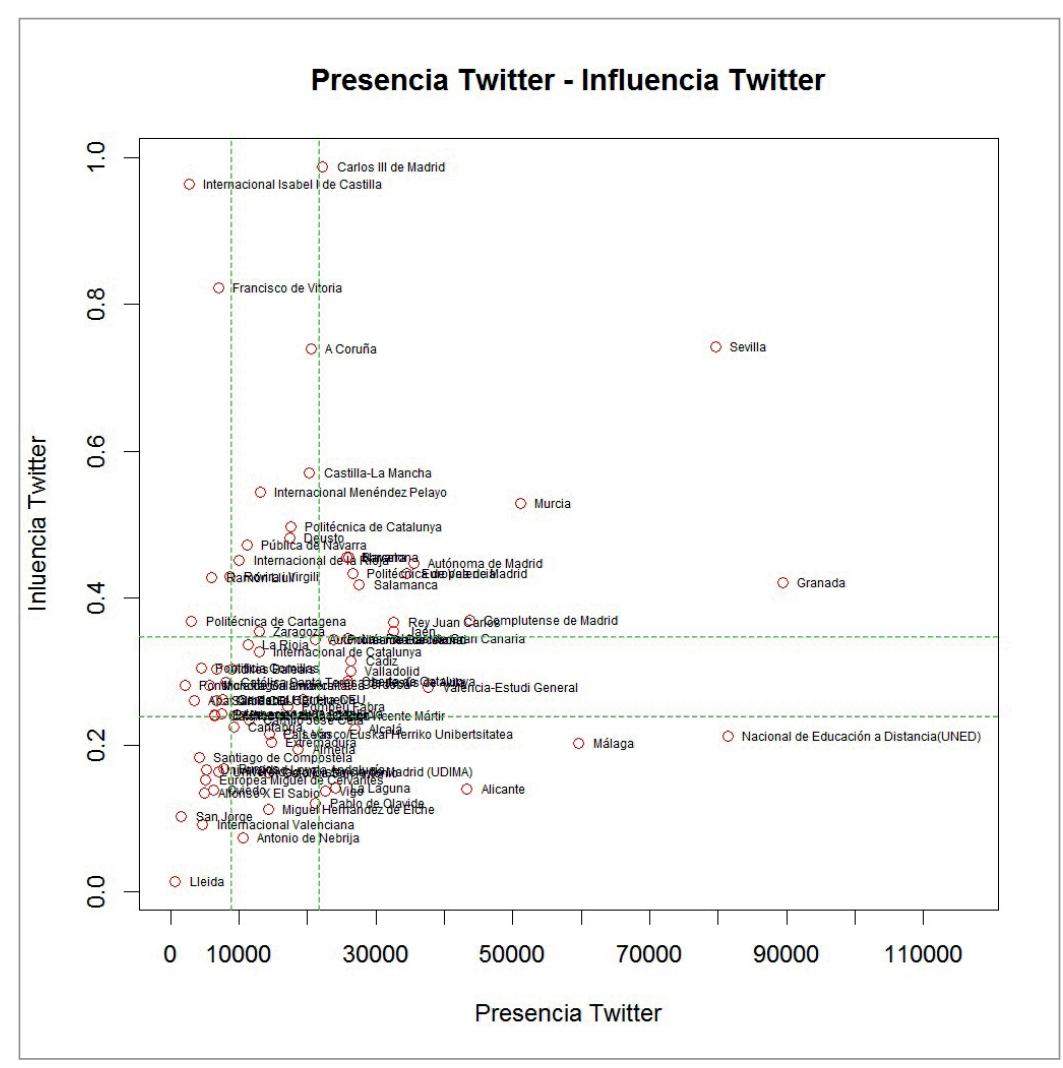

Figura 4. Gráfico de dispersión de los rankings de influencia en Twitter y Linkedln 
e Influencia global) y tres para YouTube (Presencia, Modelo de comunicación relacional e Influencia).

Por limitaciones de espacio, hemos desarrollado dos casos de estudio con datos actuales para dos de los rankings, Presencia e Influencia, en Twitter y LinkedIn. A la vista de los resultados obtenidos, hemos podido identificar que el ranking de Influencia de Twitter es el único que destaca a un grupo de universidades privadas en las primeras posiciones, aun teniendo una presencia menor que otras universidades. Los resultados asociados a la combinación de dos rankings distintos también han sido muy relevantes, mostrando por ejemplo como la Presencia y la Influencia presentan una mayor correlación en LinkedIn que en Twitter.

Este trabajo puede ayudar a los gestores universitarios a examinar su posición relativa en los distintos rankings de las RS en las que actúan y evaluar en qué medida están consiguiendo sus objetivos. En muchos casos la presencia de las universidades en RS no obedece a una estrategia de comunicación concreta e integrada con el resto de acciones de comunicación (Simón-Onieva, 2014), lo que sin duda afecta a la manera en la que actúan en las redes y a los resultados que obtienen. Son diversos los factores que pueden influir de manera positiva o negativa en el nivel de aceptación o engagement que reciben los mensajes que ponen las universidades en RS. Entre estos factores cabe destacar los formatos de contenidos utilizados, el momento de la publicación y la longitud de la misma (Cvijikj; Michahelles, 2013), a los que habría que unir el estilo de comunicación empleado centrado más en la persuasión o en el diálogo con los públicos objetivos.

\section{La Universidad Complutense de Madrid y}

la Universitat de Barcelona son dos claros ejemplos de equilibrio entre presencia e influencia en Linkedln

Como futuras líneas de trabajo nos planteamos realizar un estudio de correlación entre las posiciones que ocupan las universidades españolas en los rankings aquí presentados y su orden en otros rankings universitarios que miden otras dimensiones como los de investigación o los de docencia.

Por último, nos planteamos perfeccionar la definición de los rankings propuestos para considerar directamente el efecto del tamaño de la universidad en los valores de los indicadores. Como se indica en el informe del Ranking Issue Fundación BBVA (Pérez, 2013), el hecho de tener o no en cuenta el tamaño de las universidades a la hora de compararlas puede considerarse relevante y sería interesante analizar la relación entre el tamaño y la oferta formativa de las instituciones y su posición en nuestros rankings.

\section{Agradecimientos}

Los autores desean agradecer la ayuda del profesor Luciano Sánchez, de la Universidad de Oviedo, en la generación de los gráficos de la sección 4.3.

Este trabajo está soportado por el Ministerio de Economía y Competitividad de España bajo el proyecto NEWSOCO (ref. TIN2015-67661-P, incluyendo Fondos Europeos de Desarro-
Ilo Regional FEDER) y por el Departamento de Innovación, Ciencia y Empresa de la Junta de Andalucía bajo el Proyecto de Excelencia P12-SEJ-2592.

\section{Notas}

1. Existen países en los que estas redes no tienen tanta repercusión, como es el caso de China, que utiliza sus propias RS (Sina Weibo, QZone, Renren, Youku, etc.); Rusia, donde está muy extendida la red VK (antes VKontakte); y la India, donde Orkut era mayoritaria hasta su desaparición en 2014

2. Según la Real Academia Española, se entiende por ranking una "clasificación de mayor a menor, útil para establecer criterios de valoración".

3. También se ha presentado recientemente un estudio de presencia de las universidades españolas en otro tipo de RS más académicas y profesionales como ResearchGate y Academia.edu en González-Díaz, Iglesias-García y Codina (2015).

\section{Una Presencia alta en Twitter no implica directamente una Influencia importante en esta red social y viceversa}

\section{Bibliografía}

AIMC (2015). Estudio general de medios, 3a ola 2015 (abrilmayo).

http://www.aimc.es/-Audiencia-de-Internet-en-el-EGM-.html

Bortree, Denise S.; Seltzer, Trent (2009). "Dialogic strategies and outcomes: An analysis of environmental advocacy groups' Facebook profiles". Public relations review, v. 35, n. 3, pp. 317-319.

http://dx.doi.org/10.1016/j.pubrev.2009.05.002

Buela-Casal, Gualberto; Bermúdez, María-de-la-Paz; Sierra, Juan-Carlos; Quevedo-Blasco, Raúl; Castro, Ángel (2009). "Ranking de 2008 en productividad en investigación de las universidades públicas españolas". Psicothema, v. 21, n. 2, pp. 309-317.

http://www.ugr.es/ aepc/articulo/ranking08.pdf

Campos-Freire, Francisco; Rivera-Rogel, Diana; RodríguezHidalgo, Claudia (2014). "Presence and impact of Andean universities in online social networks". Revista latina de comunicación social, n. 69, pp. 571-592. http://dx.doi.org/10.4185/RLCS-2014-1025en

Cvijikj, Irena P.; Michahelles, Florian (2013). “Online engagement factors on Facebook brand pages". Social network analysis and mining, v. 3, n. 4, pp. 843-861.

http://cocoa.ethz.ch/downloads/2013/07/1253_10.1007_ s13278-013-0098-8.pdf

http://dx.doi.org/10.1007/s13278-013-0098-8

Del-Barrio-García, Salvador; Arquero, José-Luis; RomeroFrías, Esteban (2015). "Personal learning environments acceptance model: The role of need for cognition, e-learning satisfaction and students' perceptions". Educational technology \& society, v. 18 , n. 3, pp. 129-141.

http://digibug.ugr.es/handle/10481/37292\#.VOcoPZOLQUE 
Docampo, Domingo (2012). "Reproducibility of the Shanghai Academic Ranking of World Universities results". Scientometrics, v. 94, n. 2, pp. 567-587.

http://dx.doi.org/10.1007/s11192-012-0801-y

Docampo, Domingo; Herrera, Francisco; Luque-Martínez, Teodoro; Torres-Salinas, Daniel (2012). "Efecto de la agregación de universidades españolas en el Ranking de Shanghai $(A R W U)$ : Caso de las comunidades autónomas y los campus de excelencia". El profesional de la información, v. 21, n. 4, pp. 428-432.

http://dx.doi.org/10.3145/epi.2012.jul.16

Duncan, Tom; Moriarty, Sandra E. (1998). "A communication-based marketing model for managing relationships". Journal of marketing, v. 62, n. 2, pp. 1-13.

http://citeseerx.ist.psu.edu/viewdoc/download?doi=10.1.1. 461.5667\& $r e p=r e p 1 \&$ type $=p d f$

Gómez-Calderón, Bernardo J.; Paniagua-Rojano, Francisco-Javier (2014). "Las universidades españolas en Twitter: mensajes, contenidos y públicos". Historia y comunicación social, v. 19, Extra 1, pp. 681-694.

https://revistas.ucm.es/index.php/HICS/article/ viewFile/44994/42365

González-Díaz, Cristina; Iglesias-García, Mar; Codina, Lluís (2015). "Presencia de las universidades españolas en las redes sociales digitales científicas: caso de los estudios de comunicación". El profesional de la información, v. 24, 5, pp. 1699-2407.

http://dx.doi.org/10.3145/epi.2015.sep.12

Gonzalo-Brito, Julio; Laaser, Wolfram; Toloza, Eduardo (2012). "El uso de redes sociales por parte de las universidades a nivel institucional. Un estudio comparativo". RED. Revista de educación a distancia, n. 32, pp. 1-38.

http://www.um.es/ead/red/32/laaser_et_al.pdf

GWI (2016). GWI social. Q1 2016.

http://insight.globalwebindex.net/social

Holmberg, Kim (2015). "Online attention of universities in Finland: Are the bigger universities bigger online too?". En: Procs of ISSI 2015-15 th Int/ conf of the Int/ Society for Scientometrics and Informetrics, pp. 83-88.

http://www.issi2015.org/files/downloads/all-papers/0083. $p d f$

IAB (2015). VI Estudio redes sociales de IAB Spain. http://www.iabspain.net/wp-content/plugins/downloadmonitor/download.php?id=176

Liu, Nian C. (2015). "The story of Academic Ranking of World Universities”. International higher education, n. 54, pp. 2-3. http://ejournals.bc.edu/ojs/index.php/ihe/article/ view/8409

Merisotis, Jamie P. (2002). "On the ranking of higher education institutions". Higher education in Europe, n. 27, n. 4, pp. 361-363.

http://dx.doi.org/10.1080/0379772022000071832

O'Keeffe, Gwenn S.; Clarke-Pearson, Kathleen (2011). “The impact of social media on children, adolescents, and families". Pediatrics, v. 127, n. 4, pp. 800-804.

\section{http://dx.doi.org/10.1542/peds.2011-0054}

Ordorika, Imanol; Rodríguez-Gómez, Roberto (2010). “EI ranking Times en el mercado del prestigio universitario". Perfiles educativos, v. 32, n. 19, pp. 7-29.

http://www.scielo.org. $m x /$ scielo.php?script=sci_arttext\&pi $d=S 0185-26982010000300002$

Otto, Francis; Williams, Shirley (2014). "Official use of social network sites by African universities". En: Procs of Pan African int conf on information science, computing and telecommunications (PACT), Arusha, Tanzania, pp. 46-51. http://dx.doi.org/10.1109/SCAT.2014.7055135

Paniagua-Rojano, Francisco-Javier; Gómez-Calderón, Bernardo-José (2012). "Hacia la comunicación 2.0. El uso de las redes sociales por parte de las universidades españolas". Revista Icono14. Revista científica de comunicación y tecnologías emergentes, v. 10, n. 3

http://www.icono14.net/ojs/index.php/icono14/article/ view/473

Pérez, Francisco (dir.) (2013). Rankings Issue. http://www.u-ranking.es/analisis.php

Permatasari, Hanunm P.; Harlena, Silvia; Erlangga, Donny; Chandra, Reza (2013). "Effect of social media on website popularity: Differences between public and private universities in Indonesia". World of computer science \& information technology journal, v. 3, n. 2, pp. 32-37.

http://arxiv.org/pdf/1403.1956.pdf

Petty, Richard E.; Cacioppo, John T. (1986). The elaboration likelihood model of persuasion. New York: Springer. http://dx.doi.org/10.1007/978-1-4612-4964-1_1

Petty, Richard E.; Cacioppo, John T. (2011). Communication and persuasion: Central and peripheral routes to attitude change. Springer Science \& Business Media. ISBN: 9781 461249641

Roblyer, Margaret D.; McDaniel, Michelle; Webb, Marsena; Herman, James; Witty, James-Vince (2010). "Findings on Facebook in higher education: A comparison of college faculty and student uses and perceptions of social networking sites". The internet and higher education, v. 13, n. 3, pp. 134-140.

http://dx.doi.org/10.1016/j.iheduc.2010.03.002

Rybalko, Svetlana; Seltzer, Trent (2010). “Dialogic communication in 140 characters or less: How Fortune 500 companies engage stakeholders using Twitter". Public relations review, v. 36, n. 4, pp. 336-341.

http://dx.doi.org/10.1016/j.pubrev.2010.08.004

Simón-Onieva, José-Eduardo (2014). “El uso de las redes sociales en el ámbito de la comunicación universitaria andaluza". Revista internacional de relaciones públicas, v. 4, n. 8, pp. 139-160.

http://revistarelacionespublicas.uma.es/index.php/revrrpp/ article/view/285

The Cocktail Analysis (2015). Sexta oleada del observatorio de redes sociales.

http://es.slideshare.net/TCAnalysis/6-oleada-observatorioredes-sociales 
Tomàs-Folch, Marina; Feixas, Monica; Bernabeu, MariaDolors; Ruiz, José-María (2015). "La literatura científica sobre rankings universitarios: una revisión sistemática". REDU. Revista de docencia universitaria, v. 13, n. 3, pp. 33-54. http://red-u.net/redu/index.php/REDU/article/view/828

Torres-Salinas, Daniel; Delgado-López-Cózar, Emilio; García-Moreno-Torres, José; Herrera, Francisco (2011). "Rankings ISI de las universidades españolas según campos científicos: descripción y resultados". El profesional de la información, v. 20, n. 1, pp. 111-118.
http://recyt.fecyt.es/index.php/EPI/article/view/epi.2011. ene.14

http://dx.doi.org/10.3145/epi.2011.ene.14

Valerio, Gabriel; Herrera-Murillo, Dagoberto-José; Villanueva-Puente, Fernando; Herrera-Murillo, Natalia; Rodríguez, María-del-Carmen (2015). "En Facebook el tamaño sí importa. Engagement y el impacto de la longitud del mensaje en las fanpages de las universidades mexicanas". Revista digital universitaria, v. 12, n. 1.

http://dx.doi.org/10.7238/rusc.v12i1.1887

\section{Colección EPI Scholar}

\section{Libros científicos de Información, Documentación y Comunicación}
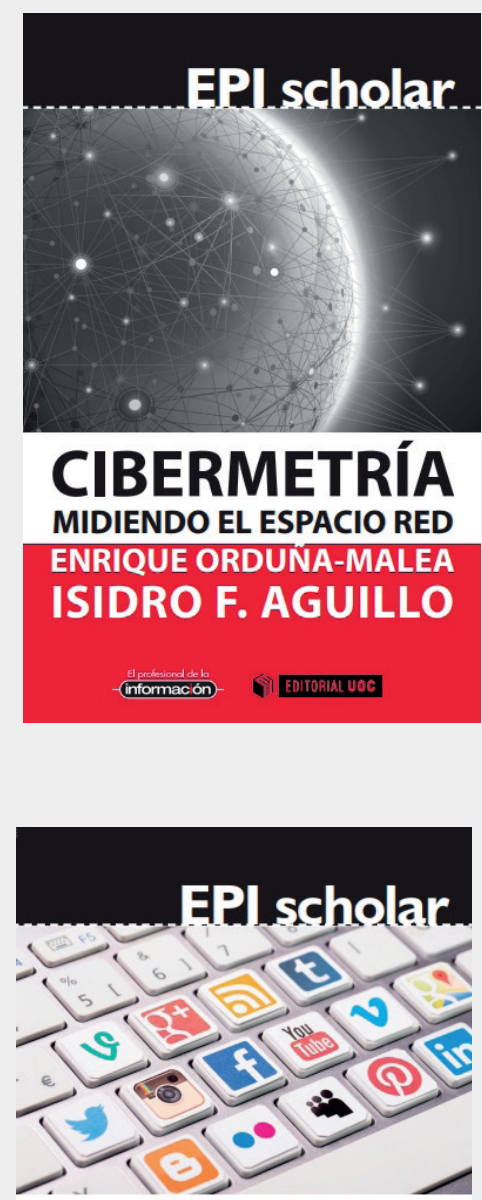

MÉTRICAS DE LA WEB SOCIAL PARA BIBLIOTECAS

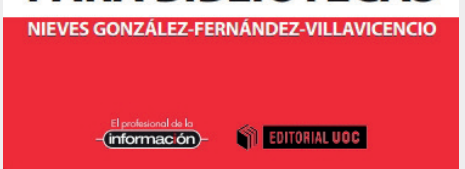

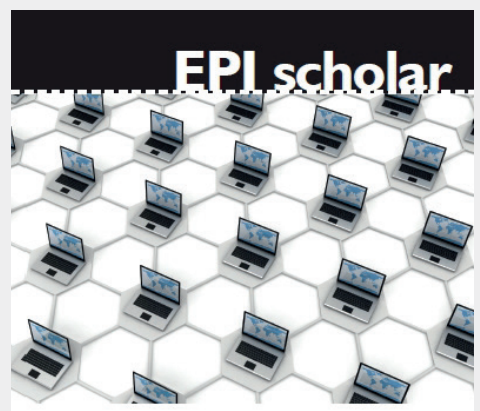

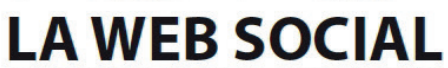
COMO NUEVO MEDIO DE COMUNICACION Y EVALUACIÓN CIENTÍFICA AMMALIA MAS-BLEDA ISIDRO F. AGUILLO

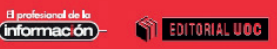

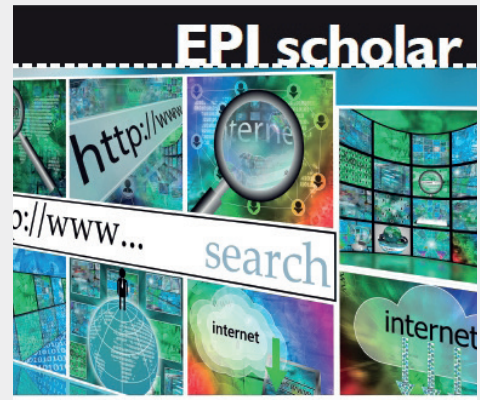

CALIDAD EN SITIOS WEB

METODO DE ANALISIS GENERAL, E-COMMERCE, IMÁGENES, HEMEROTECAS Y TURISMO RAFAEL PEDRAZA-JIMENEZ LLUISS CODINA

JAVIER GUALLAR (COORD.)

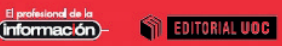

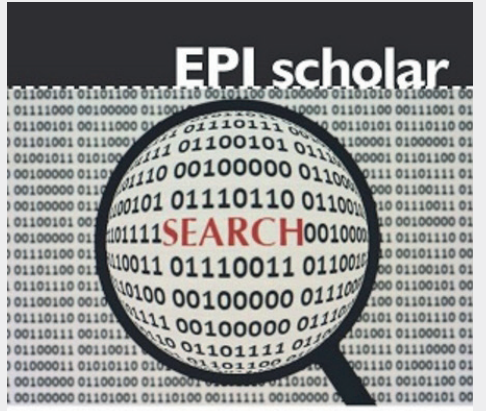

SEO

INTRODUCCIÓN A LA DISCIPLINA DEL POSICIONAMIENTO EN BUSCADORES JORGE SERRRANO-COBOS Ciformacón \& EOITORILL UOC

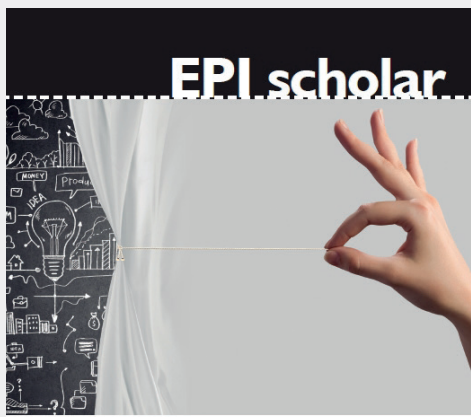

GESTIÓN DE DATOS DE INVESTIGACIÓN

FABIANO COUTO CORREA

Información: Isabel Olea epi.iolea@gmail.com http://www.elprofesionaldelainformacion.com/librosEPIScholar.html 